Tzy-Wei Hwang · Yang Kuang

\title{
Deterministic extinction effect of parasites on host populations
}

Received: 30 October 2001 / Revised version: 11 February 2002 /

Published online: 17 October 2002 - (C) Springer-Verlag 2002

\begin{abstract}
Experimental studies have shown that parasites can reduce host density and even drive host population to extinction. Conventional mathematical models for parasite-host interactions, while can address the host density reduction scenario, fail to explain such deterministic extinction phenomena. In order to understand the parasite induced host extinction, Ebert et al. (2000) formulated a plausible but ad hoc epidemiological microparasite model and its stochastic variation. The deterministic model, resembles a simple SI type model, predicts the existence of a globally attractive positive steady state. Their simulation of the stochastic model indicates that extinction of host is a likely outcome in some parameter regions. A careful examination of their ad hoc model suggests an alternative and plausible model assumption. With this modification, we show that the revised parasite-host model can exhibit the observed parasite induced host extinction. This finding strengthens and complements that of Ebert et al. (2000), since all continuous models are likely break down when all population densities are small. This extinction dynamics resembles that of ratio-dependent predator-prey models. We report here a complete global study of the revised parasite-host model. Biological implications and limitations of our findings are also presented.
\end{abstract}

\section{Introduction}

It is now widely believed that diseases and parasites were responsible for a number of extinctions on islands and on large land masses. As a result, ecologists acknowledge the importance of disease and parasites in the dynamics of population (McCallum and Dobson (1995)). Theory on the effects of parasites on host population dynamics has received much attention and focused on issues such as how the parasite induced reduction of host fecundity and survival rates change the host population dynamics, and how such dynamics be applied to predict threats to biodiversity in general and endangered species in particular. It has apparent implications

T.-W. Hwang: Department of Mathematics, Kaohsiung Normal University, 802, Kaohsiung, Taiwan, R.O.C.

Research supported by National Council of Science, Republic of China.

Y. Kuang: Department of Mathematics, Arizona state University, Tempe, AZ 85287-1804, U.S.A. e-mail: kuang@asu .edu

Work is partially supported by NSF grant DMS-0077790

Mathematics Subject Classification (2000): 34C25, 34C35, 92D25.

Key words or phrases: Microparasite model - Ratio-dependent predator-prey model - Host extinction - Global stability - Biological control 
for the use of parasites in biological control programs and for the role parasites play in driving small populations to extinction (Anderson (1982), McCallum and Dobson (1995)). A natural vehicle to study such issues is carefully formulated parasite-host interaction models. Most effort along this direction is based on the adaptations of various of classical models of epidemiology (see Ebert et al. (2000) and the extensive references cited). However, existing mathematical models for parasite-host interactions, while can address the host density reduction scenario, fail to explain host deterministic extinction phenomena.

In an effort to understand the parasite induced host extinction, Ebert et al. (2000) formulated the following plausible but ad hoc epidemiological microparasite model with horizontal transmission.

$$
\left\{\begin{array}{l}
x^{\prime}(t)=a(x+\theta y)[1-c(x+y)]-d x-b x y, \\
y^{\prime}(t)=-(d+\alpha) y+b x y \\
x(0)=x_{0}>0, y(0)=y_{0}>0,
\end{array}\right.
$$

where $x(t), y(t)$ represent the densities of uninfected (susceptible) and infected (infective) hosts at time $t$ respectively; $a$ is the maximum per capita birth rate of uninfected hosts; $\theta$ is the relative fecundity of an infected host; $c$ measures the per capita density-dependent reduction in birth rate; $d$ is the parasite-independent host background mortality; $b$ is the infection rate constant and $\alpha$ is the parasite-induced excess death rate. Notice that the disease transmission is assumed to be horizontal. Unfortunately, this SI type deterministic model predicts the existence of a globally attractive positive steady state (a rigorous proof is not given but can be easily obtained by the application of Dulac criterion with the auxiliary function $1 / x y$ and the subtle observation that $c(x(t)+y(t))<1$ for large time). Such simple dynamics clearly fails to explain the observed rich outcomes dependent on parameter values and initial population levels. In fact, a more sophisticated three dimensional nonlinear model (including elements of horizontal and vertical transmission), formulated and carefully studied earlier by Anderson (1982), also shared this failure. To see if stochastic events can lead to the extinctions of hosts, carefully designed stochastic simulation of model (1.1) was conducted be Ebert et al. (2000). The outcome indicates that extinction of host is possible in some parameter regions.

A careful examination of the above ad hoc deterministic model reveals an often misinterpreted and misunderstood model assumption. Namely, $b$, if understood as infection rate, it is the maximum number of infections an infective host can cause in a unit of time. This shows that the infection term bxy should be replaced by $b \frac{x}{x+y} y$. When the total population is constant, a fact if the disease is not fatal and the model does not address vital dynamics (the normal birth and death dynamics), then the infection term $b x y$ may be justified (since $\mathrm{b} /(\mathrm{x}+\mathrm{y})$ is now a constant) but the meaning of $b$ becomes the encounter infection rate. For large populations, individual's finite and often slow movement prevents it to make contact to a large number of individuals in a unit time. Such a mechanism is better described by $b \frac{x}{x+y} y$ than $b x y$. Conceivably, encounter infection rate makes sense only when the total population is small and steady. With this simple but important modification, model (1.1) becomes 


$$
\left\{\begin{array}{l}
x^{\prime}(t)=a(x+\theta y)[1-c(x+y)]-d x-b \frac{x y}{x+y} \\
y^{\prime}(t)=-(d+\alpha) y+b \frac{x y}{x+y} \\
x(0)=x_{0}>0, y(0)=y_{0}>0
\end{array}\right.
$$

We show that this modified model can indeed exhibit the observed parasite induced host extinction. This extinction dynamics resembles that of ratio-dependent predator-prey models (Arditi and Ginzburg (1989), Abrams and Ginzburg (2000), Arditi and Berryman (1991), Cosner et al. (1999)). Mathematically, the extinction mechanism of this and ratio-dependent population models both resulted from the degeneracy at the origin (Jost et al. (1999), Kuang and Beretta (1998), Berezovskaya, Karev and Arditi (2001), Xiao and Ruan(2001)).

Although model (1.2) exhibits the desirable deterministic extinction dynamics, we must bear in mind that to reach extinction via a continuous-time model, a population must eventually become small. In a small population, the rate of contacts with other individuals is likely to depend on the size of the population unless there is some special behavior such as an increased tendency to search for other individuals when the population density gets too low, or the population is concentrated in a small spatial region (for example, the number of contacts per unit time for each individual would remain roughly constant even if the population became quite small for populations confined to small areas such as zoos or nature reserves). In other words, when the total population becomes too small, stochastic effects become noticeable and important. In which case, the mechanism proposed and studied by Ebert et al. (2000), namely that demographic stochasticity can lead to extinction is valid. Combining the deterministic and stochastic effects, we see the plausible scenario that the deterministic dynamics brings the total population to the brink of extinction and the demographic stochasticity drives the population to extinction. From this perspective, our finding complements the work of Ebert et al. (2000).

For simplicity, we nondimensionalizes the system (1.2) with the following scaling

$$
\bar{t}=a t, \quad \bar{x}=c x, \quad \bar{y}=c y .
$$

Dropping the overlines on the variables, then the system (1.2) takes the form

$$
\left\{\begin{array}{l}
x^{\prime}(t)=(x+\theta y)[1-(x+y)]-\delta x-s \frac{x y}{x+y} \equiv F(x, y), \\
y^{\prime}(t)=-(\delta+r) y+s \frac{x y}{x+y} \equiv G(x, y), \\
x(0)=x_{0}>0, \quad y(0)=y_{0}>0
\end{array}\right.
$$

where

$$
s=\frac{b}{a}, \delta=\frac{d}{a}, r=\frac{\alpha}{a} .
$$

Observe that $\lim _{(x, y) \rightarrow(0,0)} F(x, y)=\lim _{(x, y) \rightarrow(0,0)} G(x, y)=0$. We thus define that $F(0,0)=G(0,0)=0$. Clearly, with this assumption, both $F$ and $G$ are continuous on the closure of $\mathbf{R}_{+}^{2}$ and $C^{1}$ smooth in $\mathbf{R}_{+}^{2}$ where $\mathbf{R}_{+}^{2}=\{(x, y) \mid x>$ $0, y>0\}$. So, by applying standard arguments, we see that the solutions of system (1.3) are positive, bounded and defined on $[0, \infty)$. 
Notice that ecologically meaningful initial data for (1.3) requires that $0<$ $x_{0}+y_{0}<1$. It is straightforward to see that for such initial data, $0<x(t)+y(t)<1$ for all $t>0$. Regardless of the initial population level, mathematically we show below that all positive solutions of (1.3) eventually enter and stay in the triangular positive invariant region $\Delta=\{(x, y): x>0, y>0, x+y<1\}$.

Proposition 1.1. Let $(x(t), y(t))$ be the solution of (1.3), starting at $\left(x_{0}, y_{0}\right)$ and $\left(x_{0}, y_{0}\right) \in \mathbf{R}_{+}^{2}$. Then there is a $t_{0}>0$ such that for $t>t_{0}$, we have $(x(t), y(t)) \in \Delta$.

Proof. It is straightforward to see that if $0<x_{0}+y_{0}<1$, then $0<x(t)+y(t)<1$ for all $t>0$. Moreover, it is easy to see that if there is a $t_{0}>0$, such that $0<x\left(t_{0}\right)+y\left(t_{0}\right)<1$, then $0<x(t)+y(t)<1$ for all $t>t_{0}$. Hence, we assume below that $x_{0}+y_{0}>1$. If the conclusion of the proposition is false, then $x(t)+y(t) \geq 1$ for all $t>0$. This implies that $x^{\prime}(t)<-\delta x(t)$ and hence $\lim _{t \rightarrow \infty} x(t)=0$. We see that for any $\varepsilon>0$, there is a $t_{1}>0$ such that for $t>t_{1}$, we have $0<x(t)<\varepsilon$. Then we have $y^{\prime}(t)<s \varepsilon-(\delta+r) y$. This shows that $\lim \sup _{t \rightarrow \infty} y(t) \leq s \varepsilon /(\delta+r)$. Letting $\varepsilon \rightarrow 0$, we obtain that $\lim _{t \rightarrow \infty} y(t)=0$ and therefore $\lim _{t \rightarrow \infty}(x(t)+y(t))=0<1$. This is a contradiction to $x(t)+y(t) \geq 1$ for all $t>0$, proving the proposition.

For convenience, in the rest of this paper, we assume that

(A1): $x_{0}>0, y_{0}>0$, and $x_{0}+y_{0}<1$.

Observe also that if the death rate $d$ is larger than the birth rate $a$, then $\delta>1$. We have $(x+y)^{\prime}<-(\delta-1)(x+y)$. Which implies that $\lim _{t \rightarrow \infty}(x(t), y(t))=(0,0)$. This is the intuitive outcome of the extinction of the host. In the rest of this paper, we shall assume that

(A2): $\delta \leq 1$.

In this paper we shall give a complete analysis for the asymptotic behavior of the solutions of system (1.3). The rest of this paper is organized as follows. In section 2 , by a simple but crucial change of variables, we transform the system (1.3) into a model resembles a Gause-type predator-prey system, to which a wealth of existing methods and results are applicable (Freedman (1980), Hsu et al. (2001, 2001a)). Taking advantage of this, we obtain a thorough understanding of the rich asymptotic behavior of the solutions of the system (1.3). Section 3 presents direct biological implications and limitations of our mathematical results in terms of the original parameters in system (1.2).

\section{Mathematical analysis and results}

Our objective is to gain a thorough understanding of the global dynamics of model (1.3). To this end, we need to obtain conditions that ensure the local and global stabilities of all possible steady states. Since model (1.3) is not differentiable at the origin, stability of origin can not be studied by standard linearization approach. To overcome this difficulty, we make the change of variable $(x, y) \rightarrow(u, y)$ where 
$u=x / y$ in system (1.3). This transforms it to the following system that resembles the form of a Gause-type predator-prey system.

$$
\begin{aligned}
& u^{\prime}(t)=g(u)-\varphi(u) y, \\
& y^{\prime}(t)=\psi(u) y, \\
& u(0)=u_{0}>0, y(0)=y_{0}>0
\end{aligned}
$$

where

$$
\begin{aligned}
& g(u)=(1+r-s) u+\theta \\
& \varphi(u)=(u+\theta)(u+1), \\
& \psi(u)=\frac{s u}{u+1}-(r+\delta) .
\end{aligned}
$$

Since (2.1) can also be rewritten as

$$
\begin{aligned}
& u^{\prime}(t)=\varphi(u)(h(u)-y), \\
& y^{\prime}(t)=\psi(u) y,
\end{aligned}
$$

we see that the prey isocline of the system (2.1) is given by

$$
y=\frac{g(u)}{\varphi(u)}=h(u)=\frac{(1+r-s) u+\theta}{(u+\theta)(u+1)} .
$$

Clearly, $\lim _{u \rightarrow+\infty} h(u)=0$ and

$$
h^{\prime}(u)=\left[-(1+r-s) u^{2}-2 \theta u+(r-s-\theta) \theta\right] /(u+\theta)^{2}(u+1)^{2} .
$$

From (2.4), (2.5) and some routine calculations, we have the following simple lemma which describes the qualitative behavior of the function $y=h(u)$. The proofs are omitted.

Lemma 2.1. The qualitative behaviors of $h(u)$ can be classified into the following three categories.

(a) : If $-\theta \leq s-r \leq 1$ then $h(u)>0>h^{\prime}(u)$ for all $u>0$.

(b) : If $s-r<-\theta$ then $h(u)>0$ for all $u>0$ and $h^{\prime}(u)$ has exactly one positive zero $\mu_{1}$ where $\mu_{1}=\frac{\theta+\sqrt{\theta^{2}+(1+r-s)(r-s-\theta) \theta}}{1+r-s}$.

(c) : If $s-r>1$ then $h(u)$ has exactly one positive zero $\mu_{0}$ where $\mu_{0}=\frac{\theta}{s-r-1}$ and $h^{\prime}(u)<0$ for all $u \in\left[0, \mu_{0}\right]$.

We now exam the conditions for the existences and local stabilities of boundary and positive equilibria.

System (2.1) has the boundary equilibrium $E_{1}=\left(\mu_{0}, 0\right)$ provided that $s-r>$ 1. Clearly, if $s \leq \delta+r$ then $\psi(u)<0$ for all $u>0$. On the other hand, if $s>\delta+r$ then $\psi\left(u^{*}\right)=0$ where $u^{*}=(\delta+r) /(s-\delta-r)>0$ and

$$
\psi(u)=(s-\delta-r)\left(u-u^{*}\right) /(1+u)
$$


Hence the system (2.1) has a unique positive equilibrium $E^{*}=\left(u^{*}, y^{*}\right)$ if and only if $s>\delta+r$ and $y^{*}=h\left(u^{*}\right)>0$. From (2.2), (2.3) the variational matrix of the system (2.1) is given by

$$
J(u, y)=\left[\begin{array}{cc}
\varphi^{\prime}(u)(h(u)-y)+\varphi(u) h^{\prime}(u) & -(u+\theta)(u+1) \\
s y /(1+u)^{2} & -(r+\delta)+s u /(u+1)
\end{array}\right] .
$$

The stability of equilibria $E_{1}$ and $E^{*}$ is determined by the eigenvalues of the matrix $J\left(E_{1}\right)$ and $J\left(E^{*}\right)$ respectively. The following lemma states that: whenever $E^{*}$ exists, it is asymptotically stable; if both $E^{*}$ and $E_{1}$ exist, then $E_{1}$ is a saddle; if $E^{*}$ does not exist but $E_{1}$ exists, then $E_{1}$ is asymptotically stable. A straightforward computation shows that $u^{*}>\mu_{0}$ is equivalent to $s \mu_{0} /\left(1+\mu_{0}\right)<r+\delta$. It is also equivalent to $s \theta /(s+\theta-r-1)<r+\delta$.

Lemma 2.2. For system (2.1), the following statements are true.

(a) : If $s-r \leq \delta$, then system (2.1) has no nonnegative equilibrium.

(b) : If $1<s-r$ and $u^{*}>\mu_{0}$, then $E_{1}$ is locally asymptotically stable and $E^{*}$ does not exist.

(c) : If $1<s-r$ and $u^{*}<\mu_{0}$, then $E_{1}$ is a saddle point and $E^{*}$ is locally asymptotically stable.

(d) : If $\delta<s-r \leq 1$, then $E^{*}$ is locally asymptotically stable and $E_{1}$ does not exist.

Proof. From the assumption $\delta \leq 1,(2.2)$ and (2.4), we have $g(u)>0$ and $\psi(u)<0$ for all $u>0$. Hence system (2.1) has no equilibrium point in $\mathbf{R}_{+}^{2}$ and its boundary.

For part (b) and the first part of (c), the variational matrix at $E_{1}$ is

$$
J\left(E_{1}\right)=\left[\begin{array}{cc}
\varphi\left(\mu_{0}\right) h^{\prime}\left(\mu_{0}\right) & -\varphi\left(\mu_{0}\right) \\
0 & (s-\delta-r)\left(\mu_{0}-u^{*}\right) /\left(1+\mu_{0}\right)
\end{array}\right] .
$$

Since $h^{\prime}\left(\mu_{0}\right)<0$, so if $u^{*}>\mu_{0}$ then $E_{1}$ is locally asymptotically stable. Similarly if $u^{*}<\mu_{0}$ then $E_{1}$ is a saddle point.

For part (d) and the second part of (c), from (2.6), the variational matrix at $E^{*}$ is

$$
J\left(E^{*}\right)=\left[\begin{array}{cc}
\varphi\left(u^{*}\right) h^{\prime}\left(u^{*}\right) & -\varphi\left(u^{*}\right) \\
s y^{*} /\left(1+u^{*}\right)^{2} & 0
\end{array}\right] .
$$

Since the determinant of $J\left(E^{*}\right)$ is positive and the trace of $J\left(E^{*}\right)$ is $\varphi\left(u^{*}\right) h^{\prime}\left(u^{*}\right)$. It is easy to verify that $h^{\prime}\left(u^{*}\right)<0$ under the assumptions in (c) and (d). Hence $E^{*}$ is locally asymptotically stable. Thus the assertions (c) and (d) hold.

In the following (Lemma 2.3, Theorem 2.1 and Theorem 2.2), we consider the case $s-r \leq \delta$.

Lemma 2.3. If $s-r \leq \delta$ then $\lim _{t \rightarrow \infty} u(t)=+\infty$ and $\lim _{t \rightarrow \infty} y(t)=0$.

Proof. Since $s-r \leq \delta$, then from Lemma 2.2(a), the system (2.1) has no equilibrium. 


$$
\begin{aligned}
& \text { Recall that } \mathbf{R}_{+}^{2}=\{(u, y) \mid u>0, y>0\}, \text { and } \\
& \qquad \begin{array}{l}
\Omega_{1}=\left\{(u, y) \in \mathbf{R}_{+}^{2} \mid y \geq h(u)\right\} ; \\
\Omega_{2}=\left\{(u, y) \in \mathbf{R}_{+}^{2} \mid 0<y<h(u)\right\} .
\end{array}
\end{aligned}
$$

Since there is no steady state in $\Omega_{1}$, the trajectories that start in $\Omega_{1}$ will enter $\Omega_{2}$ by crossing the $u$-isocline downward vertically. Since there is no equilibrium in $\Omega_{2}$, we must have $y(t) \rightarrow 0$ and $u(t) \rightarrow+\infty$ as $t \rightarrow+\infty$. Thus we complete the proof of the lemma.

We already know that if $\delta>1$, then $\lim _{t \rightarrow \infty}(x(t)+y(t))=0$. The next theorem shows that the same is true even if $\delta=1$, provided that $s-r \leq \delta$.

Theorem 2.1. If $s-r \leq \delta$ and $1 \leq \delta$ then the equilibrium $(0,0)$ is the global attractor for system (1.3) in $\mathbf{R}_{+}^{2}$.

Proof. From Lemma 2.3, we have $\lim _{t \rightarrow \infty} y(t)=0$ for all solutions $(x(t), y(t))$ of system (1.3). Hence, we only need to show $\lim _{t \rightarrow \infty} x(t)=0$. Assume on the contrary that there is a solution of system (1.3) such that $\lim _{\sup } \operatorname{su}_{t \rightarrow \infty} x(t)=L>0$. Now let us consider the following two cases.

Case 1. $L>\liminf _{t \rightarrow \infty} x(t) \geq 0$. There is a monotone sequence $\left\{t_{n}\right\}$ tends to $\infty$ such that $x^{\prime}\left(t_{n}\right)=0$ for all $n \geq 1$ and $\lim _{n \rightarrow \infty} x\left(t_{n}\right)=L$. Hence,

$$
0=x^{\prime}\left(t_{n}\right)=F\left(x\left(t_{n}\right), y\left(t_{n}\right)\right) \rightarrow L(1-\delta-L)<0 .
$$

So, this case is impossible.

Case 2. $L=\liminf _{t \rightarrow \infty} x(t)$. Hence, there is a sufficiently large $T$ such that $x(t)>L / 2$ and $y(t)<L(L / 2+\delta-1) / 2(\theta+1)$ for $t \geq T$. This leads to

$$
\begin{aligned}
x^{\prime}(t) & \leq x(t)(1-\delta-x(t))+\theta y(t) \\
& \leq L(1-\delta-L / 2) / 2+L \theta(L / 2+\delta-1) / 2(\theta+1) \\
& \leq-L(L / 2+\delta-1) / 2(\theta+1)<0
\end{aligned}
$$

for $t \geq T$. Thus $x(t) \rightarrow-\infty$ for $t \rightarrow \infty$. This is a contradiction and hence the lemma is proved.

The following theorem present conditions for the boundary steady state $(1-\delta, 0)$ of (1.3) to be globally attractive.

Theorem 2.2. If $s-r \leq \delta<1$ then the equilibrium $(1-\delta, 0)$ is the global attractor for system (1.3) in $\mathbf{R}_{+}^{2}$.

Proof. From Lemma 2.3, we have $\lim _{t \rightarrow \infty} \frac{x(t)}{y(t)}=+\infty$ and $\lim _{t \rightarrow \infty} y(t)=0$ for all solutions $(x(t), y(t))$ of system (1.3). Hence, for any given $\epsilon>0$, there exists a sufficiently large $T_{\epsilon}$ such that $\epsilon x(t) \geq y(t)$ for all $t \geq T_{\epsilon}$. Now from (1.3), we have

$$
\begin{aligned}
x^{\prime}(t) & =x(1-\delta-x)-(1+\theta) x y+\theta y(1-y)-s x y /(x+y) \\
& \geq x(1-\delta-x)-(1+\theta) \epsilon x^{2}-s \epsilon x /(1+\epsilon) \\
& =x(1-\delta-s \epsilon /(1+\epsilon)-(1+\epsilon+\theta \epsilon) x)
\end{aligned}
$$


for all $t \geq T_{\epsilon}$. If we choose $\epsilon$ small enough such that $1-\delta-s \epsilon /(1+\epsilon)>0$ then, by a standard comparison theorem, one obtains $\liminf _{t \rightarrow \infty} x(t)=l>0$. Let $\lim \sup _{t \rightarrow \infty} x(t)=L$. We claim that $L=l$. For otherwise, we have $L>l$ and there are two sequences $\left\{t_{n}\right\},\left\{s_{n}\right\}$ such that $x^{\prime}\left(t_{n}\right)=x^{\prime}\left(s_{n}\right)=0$ for all $n \geq 1$ and $\lim _{n \rightarrow \infty} x\left(s_{n}\right)=l, \lim _{n \rightarrow \infty} x\left(t_{n}\right)=L$. Thus

$$
L(1-\delta-L)=l(1-\delta-l)=0
$$

a contradiction. Now we have $\lim _{t \rightarrow \infty} x(t)=L$. From (1.3), we see that $x^{\prime \prime}$ is bounded on $[0, \infty)$. Hence $\lim _{t \rightarrow \infty} x^{\prime}(t)=0$. Therefore,

$$
0=\lim _{t \rightarrow \infty} x^{\prime}(t)=F(L, 0)=L(1-\delta-L) .
$$

This implies $\lim _{t \rightarrow \infty} x(t)=1-\delta$ and the theorem follows.

From now on we discuss the case $s>r+\delta$. First we consider the case $\delta<$ $s-r \leq 1$. According to Lemma 2.2 (d), $E^{*}$ is locally asymptotically stable and $E_{1}$ does not exist.

Theorem 2.3. If $\delta<s-r \leq 1$ then the positive equilibrium $E^{*}$ is the global attractor in $\mathbf{R}_{+}^{2}$ for the system (2.1).

Proof. To show that $E^{*}$ is a global attractor in $\mathbf{R}_{+}^{2}$, consider the following Lyapunov function

$$
V(u, y)=\int_{u^{*}}^{u} \frac{\psi(\xi)}{\varphi(\xi)} d \xi+\int_{y^{*}}^{y} \frac{\eta-y^{*}}{\eta} d \eta
$$

for $(u, y) \in \mathbf{R}_{+}^{2}$. Notice that $\left(u-u^{*}\right)\left(h(u)-h\left(u^{*}\right)\right) \leq 0$, which implies that $\psi(u)\left(h(u)-h\left(u^{*}\right)\right) \leq 0$. The derivative of $V$ along a solution of system (2.1) is

$$
\begin{aligned}
\dot{V}(u, y) & =(g(u)-\varphi(u) y) \psi(u) / \varphi(u)+\psi(u) y-h\left(u^{*}\right) \psi(u) \\
& =\psi(u)\left(h(u)-h\left(u^{*}\right)\right) \leq 0
\end{aligned}
$$

for $(u, y) \in \mathbf{R}_{+}^{2}$. Hence, Theorem 2.3 follows from (2.8) and the Lyapunov-LaSalle's invariance principle (Hale (1980)).

The last theorem of this paper completes the global analysis of system (2.1), and hence model (1.3).

Theorem 2.4. Let $1<s-r$ in system (2.1). The following statements are true. (a) : If $s \mu_{0} /\left(1+\mu_{0}\right)<\delta+r$ (i.e. $\left.u^{*}>\mu_{0}\right)$, then the equilibrium $E_{1}=\left(\mu_{0}, 0\right)$ of system (2.1) is the global attractor.

(b) : If $s \mu_{0} /\left(1+\mu_{0}\right)=\delta+r$ (i.e. $\left.u^{*}=\mu_{0}\right)$, then the equilibrium $E_{1}=\left(\mu_{0}, 0\right)$ of system (2.1) is the global attractor.

(c) : If $s \mu_{0} /\left(1+\mu_{0}\right)>\delta+r$ (i.e. $\left.u^{*}<\mu_{0}\right)$, then the equilibrium $E^{*}=\left(u^{*}, y^{*}\right)$ of system (2.1) is the global attractor. 
Proof. Under the assumption of the theorem, it is easy to verify that for arbitrary $\epsilon>0$, the strip

$$
\Omega_{\epsilon}=\left[0, \mu_{0}+\epsilon\right] \times[0, \infty)
$$

is positively invariant and attractive.

From the assumption of part (a), there is an $\epsilon>0$ and small enough such that $s\left(\mu_{0}+\epsilon\right) /\left(1+\mu_{0}+\epsilon\right)<\delta+r$. For this $\epsilon$, there is a $T_{\epsilon}$ large enough, such that $(u(t), y(t)) \in \Omega_{\epsilon}$ for all $t \geq T_{\epsilon}$. Thus,

$$
y^{\prime}(t)=y(s u /(1+u)-(r+\delta)) \leq\left(s\left(\mu_{0}+\epsilon\right) /\left(1+\mu_{0}+\epsilon\right)-(r+\delta)\right) y,
$$

for all $t \geq T_{\epsilon}$. This implies that $\lim _{t \rightarrow \infty} y(t)=0$.

Let $\liminf \operatorname{in}_{t \rightarrow \infty} u(t)=l$ and $\limsup _{t \rightarrow \infty} u(t)=L$. If $L>l$, then there are two sequences $\left\{t_{n}\right\},\left\{s_{n}\right\}$ such that $u^{\prime}\left(t_{n}\right)=u^{\prime}\left(s_{n}\right)=0$ for all $n \geq 1$ and $\lim _{n \rightarrow \infty} u\left(s_{n}\right)=l, \lim _{n \rightarrow \infty} u\left(t_{n}\right)=L$. Thus

$$
(1+r-s) L+\theta=(1+r-s) l+\theta=0,
$$

a contradiction. Hence, we have $\lim _{t \rightarrow \infty} u(t)=L$. From (2.1), we see that if $u^{\prime \prime}$ is bounded on $[0, \infty)$, then $\lim _{t \rightarrow \infty} u^{\prime}(t)=0$. Hence,

$$
0=\lim _{t \rightarrow \infty} u^{\prime}(t)=(1+r-s) L+\theta .
$$

This implies $\lim _{t \rightarrow \infty} u(t)=\mu_{0}$ and hence, the assertion (a).

To prove parts (b) and (c), we consider the Lyapunov function

$$
V(u, y)=\int_{u^{*}}^{u} \frac{\psi(\xi)}{\varphi(\xi)} d \xi+\int_{y^{*}}^{y} \frac{\eta-y^{*}}{\eta} d \eta
$$

for $(u, y) \in \mathbf{R}_{+}^{2}$. Notice that $\psi(u)\left(h(u)-h\left(u^{*}\right)\right) \leq 0$ for all $u \in[0, \infty)$ and if $s \mu_{0} /\left(1+\mu_{0}\right)=\delta+r$ then $E^{*}=E_{1}$. Hence, the derivative of $V$ along the solution of system (2.1) is

$$
\begin{aligned}
\dot{V}(u, y) & =(g(u)-\varphi(u) y) \psi(u) / \varphi(u)+\psi(u) y-h\left(u^{*}\right) \psi(u) \\
& =\psi(u)\left(h(u)-h\left(u^{*}\right)\right) \leq 0
\end{aligned}
$$

for $(u, y) \in \mathbf{R}_{+}^{2}$. Thus, parts (b) and (c) follow from (2.9) and the LyapunovLaSalle's invariance principle (Hale (1980)).

\section{Discussion}

A distinct feature of the simple models (1.1) and (1.2) is that the parasite population is not explicitly modeled. The general form of the model is similar to susceptible/infected (i.e. SI) models in epidemiology. A key feature of the model (1.2) is that the the term describing the infection rate is assumed to depend on the ratio of infected to susceptible individuals. In the terminology of predator-prey models, such a rate term would be called ratio-dependent. In the terminology of epidemic models 
Table 1. Complete global results of system (2.1).

\begin{tabular}{||l|l|l||}
\hline & Conditions & Results \\
\hline 1. & $\delta>1$, or $s-r \leq \delta$ plus $\delta \geq 1$ & $(0,0)$ is globally asymptotically stable. \\
\hline 2. & $s-r \leq \delta, \delta<1$ & $(1-\delta, 0)$ is globally asymptotically stable. \\
\hline 3. & $1 \geq s-r>\delta$ & $\left(x^{*}, y^{*}\right)$ is globally asymptotically stable. \\
\hline 4. & $s-r>1, s \mu_{0} /\left(1+\mu_{0}\right) \leq r+\delta$ & $(0,0)$ is globally stable. \\
\hline 5. & $s-r>1, s \mu_{0} /\left(1+\mu_{0}\right)>r+\delta$ & $\left(x^{*}, y^{*}\right)$ is globally stable. \\
\hline
\end{tabular}

such a rate term would be said to reflect proportional mixing as opposed to homogeneous mixing. The model (1.2) is derived by replacing a mass action term describing the infection rate with a ratio-dependent term in the existing model due to Ebert et al.(2000). Our analysis shows that the model may predict extinction of the host population in some cases. This behavior does not occur in models where the infection rate is described by a mass action law.

To facilitate the discussion, we first summarize the main results of system (1.3) in Table 1. The most important finding here is that the origin can be an attractor (global) for model (1.3), which may explain the often observed deterministic extinctions of hosts. There are two scenarios for this to happen: 1) $\delta \geq 1$; and 2) $s-r>1$ and $r+\delta \geq s \mu_{0} /\left(1+\mu_{0}\right)$. The first scenario is intuitive, since the death rate is no less than the birth rate which means there is no any growth in host population. Moreover, the infection reduces the birth rate and increase the death rate of the infected group which forces the total host population to decline continuously and eventually drives it to extinction.

The second scenario is more interesting. Recall that $s \mu_{0} /\left(1+\mu_{0}\right) \leq r+\delta$ is equivalent to $s \theta /(s+\theta-r-1) \leq r+\delta$. In terms of original parameters in (1.2), this condition is equivalent to

$$
b \geq \alpha+a \frac{d+\alpha-d \theta}{d+\alpha-a \theta} .
$$

If $d \leq a$ (or $\delta \leq 1$ ), then the above inequality implies $b>\alpha+a$ (or $s-r>1$ ). Observe also that this condition reduces to $\delta \geq 1$ when the disease does not exist (i.e. $\theta=1, r=0$ ). So the second scenario describes a situation when infection rate is high $(s>r+1)$ and the infection imposes a severe reduction in the birth rate of the infectious group (small $\theta$ ), or some significant yet subtle increases in the additional death rate $(\alpha)$ to the infective group in certain situations (Figure 1).

In many horizontally transmitted parasites, the parasite induced host birth rate reduction can be as high as $90 \%$, which is to say $\theta=0.1$. For these parasites, it is observed that host and parasites were prone to extinction in various experiments (p 473, Ebert et al. (2000)). The second host extinction scenario described above together with the work of Ebert et al. (2000) provides a simple and plausible explanation to such phenomena (namely, the deterministic dynamics brings the total population to the brink of extinction and the demographic stochasticity drives the 

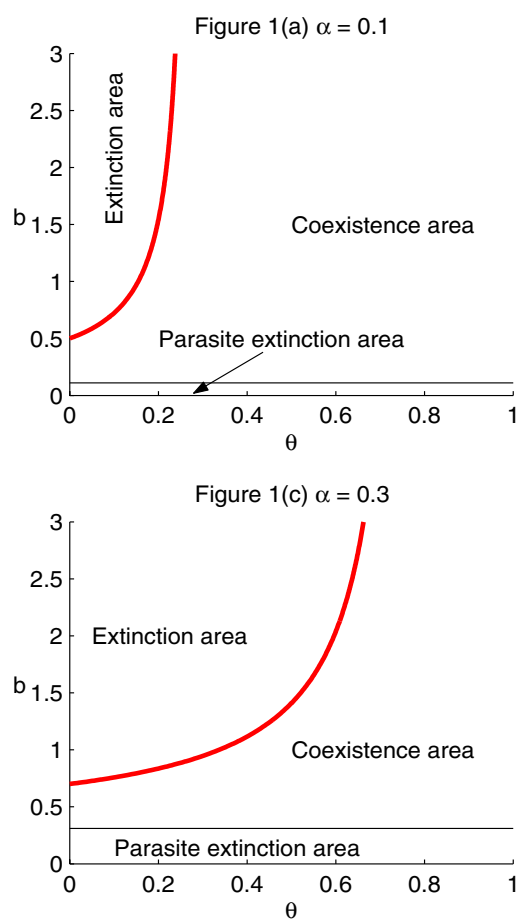

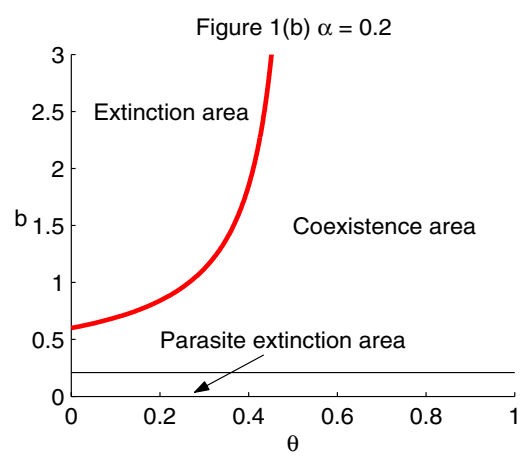

Figure $1(d) \alpha=0.4$

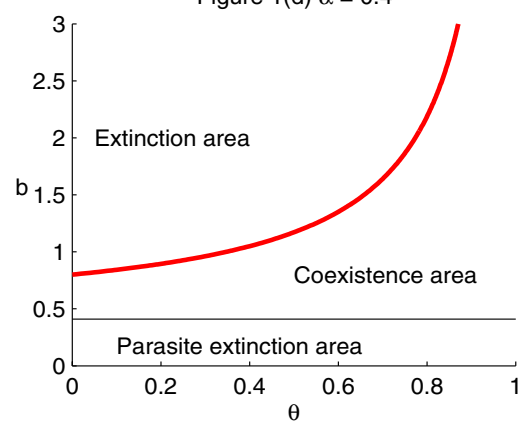

Fig. 1. In this figure, the extinction region, parasite extinction region and coexistence region for model (1.2) in $\theta b$ parameter space are depicted for $k=1, a=0.4, d=0.01$ and $\alpha=0.1,0.2,0.3,0.4$ respectively. Notice that the extinction range increases as the parasite induced mortality $\alpha$ increases and/or the parasite induced birth rate reduction $(1-\theta)$ increases.

population to extinction). In short, the field observed parasite induced deterministic host extinctions are faithfully preserved by the host extinction dynamics of the deterministic model (1.2).

The above extinction dynamics yields an unusual feature for model (1.2): even if the basic reproduction number (Hethcote (2000)) for the disease $R_{0}=b /(d+\alpha)$ is greater than 1 (equivalent to $s>r+\delta$ in model (1.3)), the disease may not persist. Instead, it may simply drive the host to extinction (when $b>\alpha+a \frac{d+\alpha-d \theta}{d+\alpha-a \theta}$ in model (1.2)).

Compare to ratio-dependent predator-prey dynamics, we see that the dynamical outcome of model (1.2) does not dependent on the initial population levels. This lack of sensitivity on initial conditions should be viewed as a limitation of the model, since in many field observation, the timing and the amount of parasite release can often alter the outcomes of the parasite-host interactions (Ebert et al. (2000)).

It is reported that there is less than $10 \%$ populations in the nature truly oscillate (Krukonis and Schaffer (1991), Kendall et al (1998)). However, when subject to human intervention, population fluctuation increases in general (Kendall et all (1998)). In addition, oscillatory population dynamics received widespread attention 
in the literature. From this perspective, another possible limitation of this simple SI type microparasite model is the lack of limit cycle behavior. Solutions typically tend to the global attractor quickly and monotonically after a brief transition period (Figure 2). The reasons cause the absence of oscillatory behaviors may due to several factors, such as 1) the particular formulation of model (1.2); the implicit assumptions that 2) the infection dynamics is instantaneous (for the importance of introduce time delayed response, see Kuang (1993) and Harrison (1995)); and that 3 ) the food resource is constant (not modeled). In reality, resources are highly dynamic and hence must be explicitly modeled in order to describe the rich dynamics of parasite-host interactions observed in the fields. This is particularly so if parasite is chosen as the control agent in a biological control process (Anderson (1982), Luck (1990)). A plausible model may build the simple infection mechanism described by model (1.2) on top of a typical predator-prey model with either prey-dependent or ratio-dependent (or the more general predator-dependent ones) functional responses. The model can take various forms depending on the specific choices of infection mechanisms and the predator functional responses. Appropriately formulated, these models shall be able to generate rich extinction dynamics such as the one documented in Hsu et al. (2002). An example of such models with prey-dependent functional response may take the form

$$
\begin{array}{rlrl}
x^{\prime}(t) & =r x\left(1-\frac{x}{K}\right)-\frac{c_{1} x(y+z)}{a+x}, & x(0)>0, \\
y^{\prime}(t) & =\frac{m_{1} x(y+f z)}{a+x}-d y-\frac{c_{2} y z}{y+z}, & y(0)>0, \\
z^{\prime}(t) & =\frac{m_{2} y z}{y+z}-(d+\alpha) z, \quad z(0)>0 .
\end{array}
$$
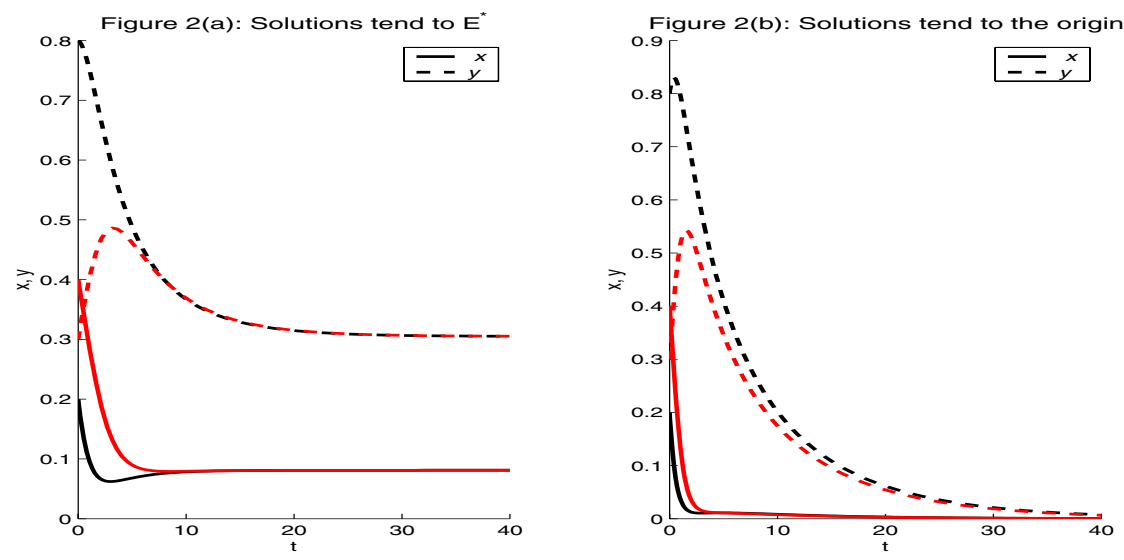

Fig. 2. In Figure 2(a), solutions tend to $E^{*}$ monotonically after a brief transition period. Here $a=0.4, d=0.01, \alpha=0.2, \theta=0.6, b=1, c=1$. From Figure $1(\mathrm{~b})$, we see that $E^{*}$ is globally asymptotically stable. In Figure 2(b), solutions tend to the origin monotonically after a brief transition period. Here $a=0.4, d=0.01, \alpha=0.2, \theta=0.2, b=2, c=1$. From Figure 1(b), we see that the origin is the global attractor. 
Here $x$ is the resource that interacts with pest. The pest is subjected to a horizontally transmitted parasite attack and is divided into two classes: the susceptible $y$ (uninfected by the parasite) and the infective $z$. It will be interesting to know if the naturally occurring ratio-dependence (resulted from the infection mechanism) in the uninfected $(y)$ and the infected pest $(z)$ equations will generate fluctuations in population levels and rich extinction dynamics that can be used together with the findings of Ebert et al. (2000) to naturally account for the various extinction scenarios observed in the fields.

Acknowledgements. The authors would like to thank Professor Ebert for his valuable comments and the referees for their insightful suggestions that improved the presentation of this manuscript.

\section{References}

[1] Abrams, P.A., Ginzburg, L.R.: The nature of predation: prey dependent, ratio-dependent or neither? Trends in Ecology \& Evolution, 15, 337-341 (2000)

[2] Arditi, R., Berryman, A.A.: The biological control paradox, Trends in Ecology \& Evolution, 6, 32 (1991)

[3] Arditi, R., Ginzburg, L.R.: Coupling in predator-prey dynamics: ratio-dependence, J. Theor. Biol., 139, 311-326 (1989)

[4] Anderson, R.M.: Theoretical basis for the use of pathogens as biological control agents of pest species, Parasitology, 84, 3-33 (1982)

[5] Berezovskaya, F., Karev, G., Arditi, R.: Parametric analysis of the ratio-dependent predator-prey model, J. Math. Biol. 43, 221-246 (2001)

[6] Cosner, C., DeAngelis, D.L., Ault, J.S., Olson, D.B.: Effects of spatial grouping on the functional response of predators, Theor. Pop. Biol., 56, 65-75 (1999)

[7] Ebert, D., Lipsitch, M., Mangin, K.L.: The effect of parasites on host population density and extinction: experimental epidemiology with Daphnia and six microparasites, American Naturalist, 156, 459-477 (2000)

[8] Freedman, H.I.: Deterministic Mathematical Models in Population Ecology, Marcel Dekker, New York, 1980

[9] Hale, J.: Ordinary Differential Equations, Krieger Publ. Co., Malabar, 1980

[10] Harrison, G.W.: Comparing predator-prey models to Luckinbill's experiment with Didinium and Paramecium, Ecology, 76, 357-374 (1995)

[11] Hethcote, H.W.: The mathematics of infectious diseases, SIAM Rev., 42, 599-653 (2000)

[12] Hsu, S.B., Hwang, T.W., Kuang, Y.: Global Analysis of the Michaelis-Menten type ratio-dependence predator-prey system, J. Math. Biol., 42, 489-506 (2001)

[13] Hsu, S.B., Hwang, T.W., Kuang, Y.: Rich dynamics of a ratio-dependent one prey two predator model, J. Math. Biol., 43, 377-396 (2001a)

[14] Hsu, S.B., Hwang, T.W., Kuang, Y.: A ratio-dependent food chain model and its applications to biological control. Math. Biosci., to appear (2002)

[15] Jost, C., Arino, O., Arditi, R.: About deterministic extinction in ratio-dependent predator-prey models. Bull. Math. Biol., 61, 19-32 (1999)

[16] Kendall, B.E., Prendergast, J., Bjornstad, O.N.: The macroecology of population dynamics: taxonomic and biogeographic patterns in population cycles. Ecol. Letters $\mathbf{1}$, 160-164 (1998) 
[17] Kendall, B.E., Briggs, C.J., Murdoch, W.W., Turchin, P., Ellner, S.P., McCauley, E., Nisbet, R.M., Wood, S.N.: Why do populations cycle? A synthesis of statistical and mechanistic modelling approaches. Ecology 80, 1789-1805 (1999)

[18] Krukonis, G., Schaffer, W.M.: Population cycles in mammals and birds: does periodicity scale with body size? Journal of Theor. Biol. 148, 469-493 (1991)

[19] Kuang, Y.: Delay Differential Equations with Applications in Population Dynamics, Academic Press, Boston, 1993

[20] Kuang, Y., Beretta, E.: Global qualitative analysis of a ratio-dependent predator-prey system, J. Math. Biol., 36, 389-406 (1998)

[21] Luck, R.F.: Evaluation of natural enemies for biological control: a behavior approach, Trends in Ecology \& Evolution, 5, 196-199 (1990)

[22] McCallum, H., Dobson, A.: Detacting disease and parasite threats to endangered species and ecosystems. Trends in Ecology \& Evolution, 10, 190-194 (1995)

[23] Xiao, D., Ruan, S.: Global dynamics of a ratio-dependent predator-prey system, J. Math. Biol., 43, 268-290 (2001) 